\title{
Classification of the regular oriented hypermaps with prime number of hyperfaces*
}

\author{
Antonio Breda d'Azevedo, Maria Elisa Fernandes \\ Center for Research and Development in Mathematics and Applications \\ Department of Mathematics, University of Aveiro, Aveiro, Portugal.
}

This paper is part of the Special Issue in Honor of the 60th Birthday of Professor Dragan Marušič

Received 30 April 2014, accepted 6 March 2015, published online 18 September 2015

\begin{abstract}
Regular oriented hypermaps are triples $(G ; a, b)$ consisting of a finite 2-generated group $G$ and a pair $a, b$ of generators of $G$, where the left cosets of $\langle a\rangle,\langle b\rangle$ and $\langle a b\rangle$ describe respectively the hyperfaces, hypervertices and hyperedges. They generalise regular oriented maps (triples with $a b$ of order 2) and describe cellular embeddings of regular hypergraphs on orientable surfaces. Previously, we have classified the regular oriented hypermaps with a prime number of hyperfaces and with no non-trivial regular proper quotients with the same number of hyperfaces (i.e. primer hypermaps with prime number of hyperfaces), which generalises the classification of regular oriented maps with prime number of faces and underlying simple graph. Now we classify the regular oriented hypermaps with a prime number of hyperfaces. As a result of this classification, we conclude that the regular oriented hypermaps with prime $p$ hyperfaces have metacyclic automorphism groups and the chiral ones have cyclic chirality groups; of these the "canonical metacyclic" (i.e. those for which $\langle a\rangle$ is normal in $G$ ) have chirality index a divisor of $n$ (the hyperface valency) and the non "canonical metacyclic" have chirality index $p$. We end the paper by counting, for each positive integer $n$ and each prime $p$, the number of regular oriented hypermaps with $p$ hyperfaces of valency $n$.
\end{abstract}

Keywords: hypermaps, maps, hypergraphs, regularity, orientably regular, chirality

Math. Subj. Class.: 05E18, 05E15, 20B25, 05C25, 05C30

\footnotetext{
* This work was supported by Portuguese funds through the CIDMA-Center for Research and Development in Mathematics and Applications, and the Portuguese Foundation for Science and Technology (FCT-Fundação para a Ciência e a Tecnologia), within project PEst-OE/MAT/UI4106/2014.

E-mail addresses: breda@ua.pt (Antonio Breda d'Azevedo), maria.elisa@ua.pt (Maria Elisa Fernandes)
} 


\section{Introduction}

Hypermaps (surface embeddings of hypergraphs), introduced by Cori [10] in 1975, have acquired great importance in recent years as a connection between permutations, extended triangle groups, Riemann surfaces, algebraic curves and Galois groups. As highlighted by Grothendieck [14], the absolute Galois group of the field of algebraic numbers acts faithfully on dessins d'enfants (hypermaps), combinatorial objects that, by Belyi's theorem [1], characterise the Riemann surfaces defined (as projective algebraic curves) over the field of algebraic numbers. The correspondence between hypermaps and Riemann surfaces is in general difficult to study, but becomes more manageable if the hypermaps are uniform (that is, if all hyperfaces have the same size $n$, all hypervertices have the same degree $k$ and all hyperedges have the same size $m$ ) and particularly better handled when they are regular.

In this paper we concentrate on regular oriented hypermaps, which are algebraically characterised by triples $\mathcal{H}=(G ; a, b)$ consisting of a finite 2-generated group $G$ and a pair $a, b$ of generators of $G$; such triples encode cellular embeddings of regular hypergraphs (bipartite graphs ${ }^{1}$ ) on compact orientable surfaces of genus

$$
g=\frac{2-(|G / l\langle b\rangle|+|G / l\langle a b\rangle|+|G / l\langle a\rangle|)}{2},
$$

where $G / l H$ stands for the left cosets of the subgroup $H$ in $G$ and $|X|$ the cardinality of $X$. The left cosets of $\langle a\rangle,\langle b\rangle$ and $\langle a b\rangle$ determine the hyperfaces, hypervertices and hyperedges of $\mathcal{H}$. Regular oriented maps are regular oriented hypermaps $(G ; a, b)$ in which the product $a b$ has order 2 .

Regular (cellular-) embeddings of graphs in orientable surfaces (regular orientable maps) have been classified for certain classes of graphs. The closest to the present paper is the classification of orientable regular embeddings of graphs of given order. This has been achieved for simple graphs of prime order [13] and of order a product of two primes [12], giving rise respectively to classifications of the regular oriented simple maps of prime order, and of order a product of two primes. Regular oriented maps of type $\{|a|,|b|\}$ are regular hypermaps of type $(|b|, 2,|a|)$. Here $|g|$ is the order of $g$.

Up to a duality a primer hypermap is a generalisation of a simple map (map with underlying simple graph). In [4] we classified the primer hypermaps with prime number of hyperfaces (left cosets of $\langle a\rangle$ in $G$ ) and now we extend the classification to regular oriented hypermaps with prime number of hyperfaces - or, by duality, to a classification of the regular oriented hypermaps with prime number of hypervertices (left cosets of $\langle b\rangle$ ) or hyperedges (left cosets of $\langle a b\rangle$ ).

There has been some contributions to the classification of regular (oriented or nonoriented) hypermaps by given number of hyperfaces; namely, on regular hypermaps (nonoriented hypermaps, which include non-orientable hypermaps and hypermaps with border) with one and two hyperfaces [9], on non-orientable regular hypermaps with a prime number of hyperfaces [19], on chiral hypermaps up to 4 hyperfaces [7], and on regular oriented hypermaps up to 5 hyperfaces [3].

This paper has five sections. The first is the actual introduction which includes two subsections, one giving a quick overview of the theory of regular oriented hypermaps and the second giving an overview of "primer" hypermaps. In this subsection we write down the most important results of [4] that are used in the third section. For a complementary

\footnotetext{
${ }^{1}$ Graphs in this paper are pseudographs, that is, they may have multiple edges, loops and free-edges.
} 
reading on these subjects we address the reader to $[16,17,11,7,5,4]$. In the second section we introduce some families of hypermaps, called "derivations", that arise from a given regular oriented hypermap, and explore their properties. The third is the classification of the regular oriented hypermaps with $p$ (prime) hyperfaces, and this will be achieved by "lifting" the "primer" hypermaps with $p$ hyperfaces classified in [4]. In the fourth section, we compute the chirality group and the "H-sequences" (an extension of type) of the regular oriented hypermaps with $p$ hyperfaces. And finally in the fifth section we compute the number of regular oriented hypermaps with $p$ hyperfaces of valency $n$.

Functions in this paper are read from right to left.

\subsection{Regular oriented hypermaps}

An (finite) oriented hypermap is a triple $\mathcal{H}=(\Omega ; a, b)$ consisting of a finite set $\Omega$ (the set of darts) and two permutations $a$ and $b$ that generate a transitive group $G$ (called the monodromy group) on $\Omega$. Hyperfaces, hypervertices and hyperedges of $\mathcal{H}$ are orbits of $\langle a\rangle$, $\langle b\rangle$ and $\langle a b\rangle$ respectively, and incidence is given by non-empty intersection of orbits. Here $a b$ means $a$ followed by $b$ since functions and actions in this paper are supposed to act from right. $\mathcal{H}$ is uniform if the permutations $a, b$ and $a b$ are regular permutations; this means that all the hyperfaces have common valency, all the hypervertices have common degree and all the hyperedges have common valency. In general we have $|\Omega| \geq|G|$. If $|\Omega|=|G|$, that is, if $G$ acts regularly on $\Omega$, then we say that $\mathcal{H}$ is a regular oriented hypermap. In such case $\Omega$ can be replaced by $G$ and the right actions of $a$ and $b$ by right multiplication. Conversely, any finite two generated group $G=\langle a, b\rangle$ determines a regular oriented hypermap $(G ; \bar{a}, \bar{b})$ where the monodromy elements $\bar{a}$ and $\bar{b}$ are the respective right permutation representations of $a$ and $b$ on $G$. The above triple describes a cellular embedding of a hypergraph $\mathcal{G}$ in an oriented surface $\mathcal{S}$ (i.e., an orientable surface with a fixed orientation). Viewing $\mathcal{G}$ as a bipartite graph, with the set of vertices partitioned into black vertices and white vertices, the hypermap $\mathcal{H}$ can be seen as a bipartite map $\mathcal{M}$ where the black vertices of $\mathcal{G}$ represent the hypervertices, the white vertices the hyperedges and the faces of $\mathcal{M}$ the hyperfaces [18]. In this representation the edges of $\mathcal{G}$ are the darts of $\mathcal{H}$ and the permutations $a$ and $b$ locally permute the darts counter clockwise $(\mathrm{CCW})$ around hyperfaces and hypervertices respectively (actually in the literature it is more common $a$ and $b$ be permutations of darts CCW around hypervertices and hyperedges, and usually denoted by $R$ and $L$ ).

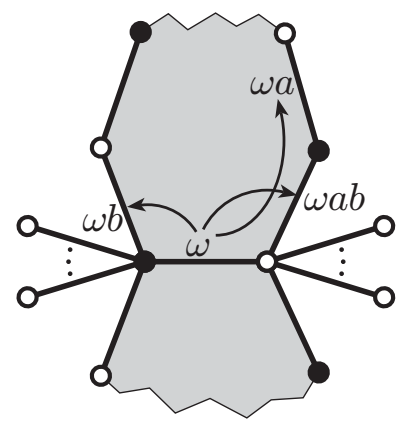

Figure 1: The effect of the permutations $a, b$ and $a b$ on a dart $\omega$.

The type of a regular oriented hypermap $\mathcal{H}$ is a triple $(k, m, n)$ where the positive integers $k=|b|, m=|a b|, n=|a|$ are the valencies of the hypervertices, hyper- 
edges and hyperfaces, in this order. An extended version of the type is the H-sequence $[k, m, n ; V, E, F ;|G|]$ where $(k, m, n)$ is the type, $V, E$ and $F$ are respectively the number of hypervertices, hyperedges and hyperfaces, and $|G|$ is the size of $G$ (or the number of darts of $\mathcal{H}$ ). The Euler characteristic of the underlying surface $\mathcal{S}$ is the characteristic of $\mathcal{H}$, and it is given by the formula $\chi=V+E+F-|G|$.

If $\mathcal{H}=(G ; a, b)$ and $\mathcal{H}^{\prime}=\left(G^{\prime} ; a^{\prime}, b^{\prime}\right)$ are two regular oriented hypermaps, then $\mathcal{H}$ covers $H^{\prime}$ if the assignment $a \mapsto a^{\prime}, b \mapsto b^{\prime}$ can be extended to a (canonical) epimorphism of monodromy groups $G \rightarrow G^{\prime}$. The hypermap $\mathcal{H}$ is isomorphic to $\mathcal{H}^{\prime}, \mathcal{H} \cong \mathcal{H}^{\prime}$, if the canonical epimorphism $G \mapsto G^{\prime}$, is an isomorphism. A hypermap is reflexible if it is isomorphic to its mirror image $\overline{\mathcal{H}}=\left(G ; a^{-1}, b^{-1}\right)$, otherwise it is chiral. The chirality group of $\mathcal{H}$ is the smallest normal subgroup $X(\mathcal{H})$ of $G$ such that $\mathcal{H} / X(\mathcal{H})$ is reflexible. This group ranges from $X(\mathcal{H})=1$, when $\mathcal{H}$ is reflexible, to $X(\mathcal{H})=\operatorname{Mon}(\mathcal{H})$ when $\mathcal{H}$ is totally chiral [5, 6]. The Chirality index of $\mathcal{H}$ is the size $\kappa=\kappa(\mathcal{H})=|X(\mathcal{H})|$.

Let $\Delta$ denote the free product $C_{2} * C_{2} * C_{2}$ generated by $r_{0}, r_{1}$ and $r_{2}$, and $\Gamma$ be the normal subgroup of index 2 in $\Delta$ generated by $a=r_{0} r_{1}$ and $b=r_{1} r_{2}$, a free group of rank 2. Any regular oriented hypermap $\mathcal{H}$ corresponds an unique normal subgroup $H$ in $\Gamma$, called the fundamental hypermap subgroup, such that $\mathcal{H} \cong(\Gamma / H ; H a, H b)$. In this context the chirality group of $\mathcal{H}$ is given by $X(\mathcal{H})=H \bar{H} / H$, where $\bar{H}=H^{r_{1}}$. If $\langle a, b \mid R(a, b)\rangle$ is a presentation of the monodromy group $G$, where $R(a, b)$ denotes a set of relators on $a$ and $b$, then the chirality group of $\mathcal{H}$ is $X(\mathcal{H})=\left\langle R\left(a^{-1}, b^{-1}\right)\right\rangle^{G}$, the normal closure in $G$ of the subgroup generated by $R\left(a^{-1}, b^{-1}\right)$ [2].

The regular oriented hypermaps $\mathcal{H}=(G ; a, b)$ with 1 and 2 hyperfaces are all reflexible and the chiral hypermaps with 3 and 4 hyperfaces are all (face-)canonical metacyclic, that is, the monodromy group $G$ is the metacyclic group $\left\langle a, b \mid a^{n}=1, b^{m}=a^{s}, b a b^{-1}=a^{t}\right\rangle$ with $(t-1) s=0 \bmod n$ and $t^{m}=1 \bmod n$. Equally we say that $(G ; a, b)$ is vertexcanonical (resp. edge-canonical) metacyclic if $\langle b\rangle$ (resp. $\langle a b\rangle$ ) is normal in $G$. $\mathcal{H}$ is vertex-canonical (resp. edge-canonical) metacyclic if and only if $\mathcal{H} \delta_{1}$ (resp. $\mathcal{H} \delta_{0}$ ) is facecanonical metacyclic, where $\delta_{1}$ is the dual operation $a \mapsto b^{-1}, b \mapsto a^{-1}$ that transpose hypervertices with hyperfaces, and $\delta_{0}$ is the dual operation $a \mapsto a b, b \mapsto b^{-1}$ that transpose hyperedges with hyperfaces. Another dual operation is the mirror operation $\mu: a \mapsto a^{-1}$, $b \mapsto b^{-1}$ that maps $\mathcal{H}$ to its mirror image $\mathcal{H} \mu=\overline{\mathcal{H}}$. Face-, vertex- and edge-canonical metacyclic hypermaps have cyclic chirality groups with chirality index $\frac{n}{\operatorname{gcd}\left(n, t^{2}-1\right)}$; while the chirality group of a face-canonical hypermap is generated by $a^{t^{2}-1}$ [7], the chirality group of a vertex- or edge-canonical metacyclic hypermap is generated by $\left(a^{t^{2}-1} \delta_{1}\right)^{-1}=$ $b^{t^{2}-1}$ or by $a^{t^{2}-1} \delta_{0}=(a b)^{t^{2}-1}$, respectively [8, Lemma 2.1]. Therefore a (face-, vertexor edge-) canonical metacyclic hypermap is chiral if and only if $t^{2} \neq 1 \bmod n$.

By canonical metacyclic we just mean face-canonical metacyclic.

In contrast, most of the hypermaps appearing in the classification [3] are not canonical metacyclic. However, we will show that all regular oriented hypermaps with a prime number of hyperfaces have metacyclic automorphism groups, though not necessarily being (face-, vertex- or edge-) canonical metacyclic hypermaps.

\subsection{Primer hypermaps}

We can use the equivalence of Proposition 11 of [4] for our definition of primer hypermap as it gives a good general idea behind the concept. A (face-) primer hypermap is a regular oriented hypermap with no non-trivial regular proper quotients with the same number of 


\section{hyperfaces.}

Any regular hypermap $\mathcal{H}=(G ; a, b)$ covers a unique primer hypermap $\mathcal{P}=\mathcal{P}(\mathcal{H})$. In particular the hyperface valency $l$ of its primer hypermap divides the hyperface valency $n$ of $\mathcal{H}$. For consistency we reserve the letter $\ell$ to denote the valency of a hyperface of a primer hypermap and set aside the letter $n$ for the valency of a hyperface of a non necessarily primer hypermap.

This primer hypermap can be constructed in the following way. When the elements of $G$ act on $G$ (set of darts) on the right they act as monodromy elements, but when they act on the left they act as automorphisms of $\mathcal{H}$. Therefore each element $\gamma \in G$ induces an automorphism $\varphi_{\gamma}: g \mapsto \gamma g$ of $\mathcal{H}$. In particular the automorphisms $\varphi_{a}: g \mapsto a g$ and $\varphi_{b}: g \mapsto b g$, induced by $a$ and $b$, correspond to one-step global counter-clockwise rotations about the hyperface and the hypervertex (respectively) that contain the identity dart. Since $\mathcal{H}$ is regular we have $A u t(\mathcal{H})=\left\langle\varphi_{a}, \varphi_{b}\right\rangle \cong G$ and since functions in this paper act on the right, $\varphi_{\gamma_{1} \gamma_{2}}=\varphi_{\gamma_{2}} \varphi_{\gamma_{1}}$ and thus $\varphi_{\left(\gamma_{1} \gamma_{2}\right)^{-1}}=\varphi_{\gamma_{1}^{-1}} \varphi_{\gamma_{2}^{-1}}$, and so,

$$
\mathcal{H}=(G ; a, b) \cong\left(A u t(\mathcal{H}) ;\left(\varphi_{a}\right)^{-1},\left(\varphi_{b}\right)^{-1}\right) .
$$

The action of $A u t(\mathcal{H})$ on $\mathcal{H}$ induces a transitive action of $A u t(\mathcal{H})$ on the set of the hyperfaces $\mathcal{F}=G / l\langle a\rangle$ of $\mathcal{H}$, where the symbol $G / l K$ represents the left cosets of $K$ in $G$. Under this action, each $\varphi_{\gamma} \in A u t(\mathcal{H})$, or equivalently each $\gamma \in G$, determines a permutation $\pi_{\gamma} \in \operatorname{Sym}(\mathcal{F})$ defined by $g\langle a\rangle \mapsto \gamma g\langle a\rangle$. In particular, the automorphisms $\varphi_{a}$ and $\varphi_{b}$ give rise to permutations $A=\pi_{a}{ }^{-1}$ and $B=\pi_{b}{ }^{-1}$ on $\mathcal{F}$. Labelling the hyperfaces of $\mathcal{H}$ by $1,2, \ldots, F$, the permutations $A$ and $B$ are elements of the symmetric group $S_{F}$. Let $P$ be the subgroup of $S_{F}$ generated by $A$ and $B$. Then $\mathcal{P}=\mathcal{P}(\mathcal{H})=(P ; A, B)$ is the primer hypermap determined by $\mathcal{H}$. The subgroup $P$ of $S_{F}$ generated by $A$ and $B$ is called the (face-) primer group of $\mathcal{H}$. We note that we are not adopting the notation $\mathcal{P}=\left(P ; A^{-1}, B^{-1}\right)$ we have used in [4].

The function $\Pi: G \longrightarrow P, \gamma \mapsto \gamma \Pi=\pi_{\gamma}{ }^{-1}=\pi_{\gamma^{-1}}$ which maps $a \mapsto A$ and $b \mapsto B$, is an epimorphism with kernel $\operatorname{Kern}(\Pi)=\left\langle a^{|A|}\right\rangle$ (Proposition 5 of [4]). Therefore it induces an epimorphism $\Pi: \mathcal{H} \longrightarrow \mathcal{P}$ branched over hyperfaces. Moreover, since $P \cong G /\left\langle a^{|A|}\right\rangle$, we get:

Corollary 7 [4]: for any word $r(A, B)$ on $A, B, r(A, B)=1$ if and only if $r(a, b)=a^{u}$ for some $u=0 \bmod |A|$ ".

To recognise a canonical metacyclic hypermap from its primer we have the following proposition:

Proposition 4 [4]: $\mathcal{H}$ is (face-) canonical metacyclic if and only if $A=1$.

The chirality group of the primer hypermap is a factor group of the chirality group of the hypermap (Proposition 9 of [4]), that is, $X(\mathcal{P}(\mathcal{H}))=X(\mathcal{H}) / K$ for some $K$. Consequently, the chirality index $\kappa(\mathcal{P}(\mathcal{H}))$ divides $\kappa(\mathcal{H})$. Hence if $\mathcal{P}(\mathcal{H})$ is chiral then also $\mathcal{H}$ is chiral. The converse is not true.

The main theorem of [4] says:

Theorem 16 [4]: $\mathcal{P}$ is a primer hypermap with $p$ (prime) hyperfaces (each of valency $\ell$ ) if and only if $\mathcal{P} \cong \mathcal{P}_{k}^{p, \ell, t}=\left(M(p, \ell, 0, t) ; y, x y^{k}\right)$ for some $\ell, t \in\{1, \ldots, p-1\}$ and $k \in\{0, \ldots, \ell-1\}$ such that: (1) $\ell$ is a divisor of $p-1$, (2) $t^{\ell}=1 \bmod p$, (3) if $\ell>1, \quad t^{i} \neq 1 \bmod p$ for each $i \in\{1,2, \ldots, \ell-1\}$. Here $M(p, \ell, 0, t)$ is the metacyclic group $\left\langle x, y \mid x^{p}=y^{\ell}=1, x^{y}=x^{t}\right\rangle=\langle x\rangle \rtimes\langle y\rangle$. Different parameters $k$, $\ell$ and $t$ correspond to non-isomorphic primer hypermaps with p hyperfaces of valency $\ell$. 
Note that if $\mathcal{H}$ is a regular oriented hypermap with $p$ (not necessarily prime) hyperfaces, each of valency $n$, then $\mathcal{H}$ covers a unique primer hypermap $\mathcal{P}$ with $p$ hyperfaces, each of valency $\ell$ with $\ell$ dividing $n$. Also useful is the following corolloary:

Corollary 17 [4]: The H-sequences of the primer hypermaps $\mathcal{P}_{k}^{p, \ell, t}$ given above are

(1) $[p, p, 1 ; 1,1, p ; p]$ if $k=0$ and $\ell=1 \quad(\Rightarrow t=1)$;

(2) $[p, \ell, \ell ; \ell, p, p ; \ell p]$ if $k=0$ and $\ell>1 \quad(\Rightarrow p>2)$;

(3) $[\ell, p, \ell ; p, \ell, p ; \ell p]$ if $k=\ell-1>0 \quad(\Rightarrow p>2)$;

(4) $\left[\frac{\ell}{(\ell, k)}, \frac{\ell}{(\ell, k+1)}, \ell ; p(\ell, k), p(\ell, k+1), p ; \ell p\right]$ if $0<k<\ell-1 \quad(\Rightarrow p>2)$,

where, for space saving, $(u, v)$ stands for $\operatorname{gcd}(u, v)$, the greatest common divider of $u$ and $v$.

\section{Derivations}

Before we start with the classification, we introduce several families of regular oriented hypermaps that are derived from a given hypermap. These families together with their properties will be useful later on.

Let $\mathcal{H}=(G ; a, b)$ be a regular (oriented) hypermap with $F$ hyperfaces of valency $n$. The following regular hypermaps, which we call derivations of $\mathcal{H}$, all have the same number of hyperfaces $F$, and the same hyperface-valency $n$.

(1) The mirror $\overline{\mathcal{H}}=\left(G ; a^{-1}, b^{-1}\right)$;

(2) The mid-mirror $M m(\mathcal{H}):=\left(G ; a, b^{-1}\right)$;

(3) The $k$-Left family $L_{k}(\mathcal{H}):=\left(G ; a, a^{k} b\right)$, for each $k \in\{1, \ldots, n-1\}$,

(4) The $k$-Right family $R_{k}(\mathcal{H}):=L_{k}(\mathcal{H})^{a^{k}}=\left(G ; a^{a^{k}},\left(a^{k} b\right)^{a^{k}}\right)=\left(G ; a, b a^{k}\right)$, and

(5) The $(0,1)$-dual $D_{(0,1)}(\mathcal{H})=M m\left(L_{1}(\mathcal{H})\right)=\left(G ; a,(a b)^{-1}\right)$; this is the hypermap resulting from $\mathcal{H}$ by swapping hypervertices with hyperedges.

One easily sees that $\overline{\overline{\mathcal{H}}}=\mathcal{H}, \quad M m(M m(\mathcal{H}))=\mathcal{H}, D_{(0,1)}\left(D_{(0,1)}(\mathcal{H})\right)=\mathcal{H}$, $L_{n-k}\left(L_{k}(\mathcal{H})\right)=L_{k}\left(L_{n-k}(\mathcal{H})\right)=\mathcal{H}$ and $R_{n-k}\left(R_{k}(\mathcal{H})\right)=R_{k}\left(R_{n-k}(\mathcal{H})\right)=\mathcal{H}$.

Let $\mathcal{D}(\mathcal{H})$ denote one of the derivations of $\mathcal{H}$. Then $\mathcal{D}$ defines an operation $\mathcal{D}: \mathcal{H} \mapsto$ $\mathcal{D}(\mathcal{H})$ that takes a regular oriented hypermap with $F$ hyperfaces of valency $n$ to a regular oriented hypermap with $F$ hyperfaces of valency $n$. This operation has the inverse defined by

$$
\mathcal{D}^{-1}= \begin{cases}\mathcal{D} & \text { if } \mathcal{D} \text { is the mirror, mid-mirror or the }(0,1) \text {-dual } \\ L_{n-k} & \text { if } \mathcal{D}=L_{k} \\ R_{n-k} & \text { if } \quad \mathcal{D}=R_{k} .\end{cases}
$$

Denote by $\Pi, \bar{\Pi}, \Pi_{M}, \Pi_{L}$ and $\Pi_{R}$ the corresponding homomorphisms $G \longrightarrow S_{p}$, $\gamma \mapsto \pi_{\gamma^{-1}}$. For example, $\Pi: a \mapsto \pi_{a^{-1}}, b \mapsto \pi_{b^{-1}}$ and $\Pi_{L}: a \mapsto \pi_{a^{-1}}, a^{k} b \mapsto \pi_{b^{-1} a^{-k}}$. As $b \Pi_{L}=\left(a^{-k} a^{k} b\right) \Pi_{L}=a^{-k} \Pi_{L} a^{k} b \Pi_{L}=\pi_{a^{k}} \pi_{b^{-1} a^{-k}}=\pi_{b^{-1} a^{-k} a^{k}}=\pi_{b^{-1}}$, then $\Pi_{L}=\Pi$. Similarly we have $\Pi=\bar{\Pi}=\Pi_{L}=\Pi_{M}$. Since the primer hypermap of $\mathcal{H}$ is $\mathcal{P}(\mathcal{H})=(G \Pi ; a \Pi, b \Pi)$ and $\mathcal{H}$ is primer if and only if $\operatorname{Ker}(\Pi)=1$, we immediately have,

Proposition 2.1. Let $\mathcal{H}$ be a regular hypermap and $\mathcal{P}(\mathcal{H})$ be its primer hypermap. Then 
(1) $\mathcal{P}(\mathcal{D}(\mathcal{H}))=\mathcal{D}(\mathcal{P}(\mathcal{H})$ ), for any derivation $\mathcal{D}$ of $\mathcal{H}$.

(2) $\mathcal{H}$ is primer if and only if any of its derivations is primer.

Let $\mathcal{F}_{\mathcal{P}}$ denote the family of regular hypermaps with primer hypermap $\mathcal{P}$. As an immediate consequence of above, $\mathcal{D}\left(\mathcal{F}_{\mathcal{P}}\right)=\mathcal{F}_{\mathcal{D}(\mathcal{P})}$, and, as a consequence of this, we have $\mathcal{D}(\mathcal{H}) \in \mathcal{F}_{\mathcal{P}} \Leftrightarrow \mathcal{H} \in \mathcal{F}_{\mathcal{D}^{-1}(\mathcal{P})}$.

Let $\mathcal{H}=(G ; a, b)$ be a regular hypermap and $\langle a, b \mid R(a, b)\rangle$ be a presentation for the monodromy group $G$. Let $x=X(a, b)$ and $y=Y(a, b)$ be another pair of generators of $G$. Then the original generators $a$ and $b$ can be written as words in $x$ and $y$, say $a=A(x, y)$ and $b=B(x, y)$.

Proposition 2.2. If the change of generators a to $x$ and $b$ to y produce no extra relations, that is, if $x=X(A(x, y), B(x, y))$ and $y=Y(A(x, y), B(x, y))$ are not new relations, and there is a $w \in G$ such that the conjugations $A^{w}=w^{-1} A w$ and $B^{w}=w^{-1} B w$ coincide with their inverse order words, in symbols $A^{w}=\overleftarrow{A}$ and $B^{w}=\overleftarrow{B}$, then both hypermaps $\mathcal{H}=(G ; a, b)$ and $\mathcal{Q}=(G ; x, y)$ have the same chirality group.

Proof. The non-existence of any extra relations implies that $\langle x, y \mid R(A(x, y), B(x, y))\rangle$ is another presentation of $G$, this time as a function of the new generators $x$ and $y$. By Theorem 1 of [2] we have,

$$
\begin{aligned}
X(\mathcal{Q}) & =\left\langle R\left(A\left(x^{-1}, y^{-1}\right), B\left(x^{-1}, y^{-1}\right)\right)\right\rangle^{G}=\left\langle R\left(\overleftarrow{A}(x, y)^{-1}, \overleftarrow{B}(x, y)^{-1}\right)\right\rangle^{G} \\
& =\left\langle R\left(A^{w}(x, y)^{-1}, B^{w}(x, y)^{-1}\right)\right\rangle^{G} \\
& =\left\langle R\left(A(x, y)^{-1}, B(x, y)^{-1}\right)\right\rangle^{G}=\left\langle R\left(a^{-1}, b^{-1}\right)\right\rangle^{G} \\
& =X(\mathcal{H}) .
\end{aligned}
$$

We saw in Proposition 2.1 that $\mathcal{H}$ is primer if and only if any of its derivations $\mathcal{D}(\mathcal{H})$ is also primer. Now we show that this is also true for chirality.

Corollary 2.3. Let $\mathcal{D}$ be a derivation of $\mathcal{H}$. Then $X(\mathcal{D}(\mathcal{H}))=X(\mathcal{H})$; that is $\mathcal{H}$ and its derivations $D(\mathcal{H})$ all share the same chirality group. In particular, if some derivation of $\mathcal{H}$ is chiral then also $\mathcal{H}$ is chiral.

Proof. In Proposition 2.2 take $w=i d$ if $\mathcal{Q}=\overline{\mathcal{H}}$ or $M m(\mathcal{H})$, and take $w=a^{-k}$ if $\mathcal{Q}=L_{k}(\mathcal{H})$ or $R_{k}(\mathcal{H})$.

Consider the families $\mathcal{P}_{I}^{p}=\left\{\mathcal{P}_{0}^{p, 1,1}\right\}, \mathcal{P}_{I I}^{p}=\left\{\mathcal{P}_{0}^{p, \ell, t}\right\}_{\ell, t}$ with $\ell>1, \mathcal{P}_{I I I}^{p}=\left\{\mathcal{P}_{\ell-1}^{p, \ell, t}\right\}_{\ell, t}$ with $\ell>1$, and $\mathcal{P}_{I V}^{p}=\left\{\mathcal{P}_{k}^{p, \ell, t}\right\}_{k, \ell, t}$ with $\ell>1$ and $0<k<\ell-1$, of the primer hypermaps with H-sequences (1), (2), (3) and (4) respectively, of Corollary 17 [4]. Then $\mathcal{P}_{I I I}^{p}=$ $R_{\ell-1}\left(\mathcal{P}_{I I}^{p}\right)$ and $\mathcal{P}_{I V}^{p}=R_{k}\left(\mathcal{P}_{I I}^{p}\right)$. Taking into account Corollary 2.3 and [7, Corollary 9] for the chirality group of canonical metacyclic hypermap, we have the following result shown in [4]:

Corollary 2.4. If $\mathcal{P}=\mathcal{P}_{k}^{p, \ell, t}=\left(G ; y, x y^{k}\right)$ is a primer hypermap with $p$ hyperfaces $(p$ prime) then

$$
X(\mathcal{P})= \begin{cases}1 \text { (reflexible) } & \text { if } \mathcal{P} \in \mathcal{P}_{I}^{p} \\ \left\langle y^{t^{2}-1}\right\rangle & \text { if } \mathcal{P} \in \mathcal{P}_{I I}^{p}, \mathcal{P}_{I I I}^{p} \text { or } \mathcal{P}_{I V}^{p}\end{cases}
$$




\section{The classification}

Let $p$ be a prime number. We now proceed with the classification of the regular oriented hypermaps with $p$ hyperfaces. Let $\mathcal{H}=(G ; a, b)$ be a regular oriented hypermap with $p$ hyperfaces (of valency $n$ ) and $\mathcal{P}=(G \Pi ; a \Pi, b \Pi)=(P ; A, B)$ be its primer hypermap, which also has $p$ hyperfaces. In what follows,

$$
M(n, p, u, t):=\left\langle a, b \mid a^{n}=1, b^{p}=a^{u}, b^{-1} a b=a^{t}\right\rangle
$$

is the metacyclic group with parameters $n, p, u, t$, and

$$
G_{n, u, v}^{p, \ell, t}:=\left\langle a, b \mid a^{n}=1, b^{p}=a^{u},\left[a^{\ell}, b\right]=1, b a b^{-t}=a^{v}\right\rangle .
$$

Before we state and prove the main theorem, we first prove the following lemma,

Lemma 3.1. Let $G=\langle a, b\rangle$, $p$ an odd prime and $t, \ell$ positive integers such that $t \neq$ $1 \bmod p, t^{\ell}=1 \bmod p$ and $p=1 \bmod \ell$. If (i) $b^{p} \in\left\langle a^{\ell}\right\rangle, \quad$ (ii) $b^{i} a b^{-i t} \in\left\langle a^{\ell}\right\rangle a$, for any $i=1,2, \ldots, p$, then $b \leftrightharpoons a^{\ell}$, where the symbol means "commutes with".

Proof. As $t^{\ell}=1 \bmod p$, by (i) we have $b^{t^{\ell}-1} \in\left\langle a^{\ell}\right\rangle$. On the other hand, taking $i=$ $1, t, t^{2}, \ldots, t^{\ell-1}$, (ii) yields the following information $b a b^{-t}, b^{t} a b^{-t^{2}}, \ldots, b^{t^{\ell-1}} a b^{-t^{\ell}} \in$ $\left\langle a^{\ell}\right\rangle a$. Multiplying the first $i$ of these words (in the same order as shown) and the last $\ell-i$ words we get $b a^{i} b^{-t^{i}} \in\left\langle a^{\ell}\right\rangle a^{i}$ and $b^{t^{i}} a^{\ell-i} b^{-t^{\ell}} \in\left\langle a^{\ell}\right\rangle a^{\ell-i}$, respectively. Then $b a^{\ell} b^{-t^{\ell}} \in\left\langle a^{\ell}\right\rangle$ and thus

$$
b a^{\ell} b^{-1}=\left(b a^{\ell} b^{-t^{\ell}}\right) b^{t^{\ell}-1}=a^{V}
$$

for some $V=0 \bmod \ell$. On the other hand,

$$
b^{t^{i}} a^{\ell} b^{-t^{i}}=\left(b^{t^{i}} a^{\ell-i} b^{-1}\right)\left(b a^{i} b^{-t^{i}}\right)=\left(b a^{i} b^{-t^{i}}\right)\left(b^{t^{i}} a^{\ell-i} b^{-1}\right)=b a^{\ell} b^{-1}=a^{V}
$$

for each $i \in\{0, \ldots, \ell-1, \ell\}$. Consequently, $b^{\left(t^{i}-1\right)} a^{\ell} b^{1-t^{i}}=b^{-1} a^{V} b=a^{\ell}$ for every integer $i \in\{0, \ldots, \ell-1, \ell\}$. Taking $i=\ell$ we get

$$
\left[a^{\ell}, b^{t^{i}-1}\right]=1, \quad i=0, \ldots, \ell
$$

In particular,

$$
b^{(t-1)+\left(t^{2}-1\right)+\ldots+\left(t^{\ell-1}-1\right)}=b^{\left(1+t+t^{2}+\ldots+t^{\ell-1}\right)-\ell} \leftrightharpoons a^{\ell} .
$$

As $t \neq 1 \bmod p$ and $t^{\ell}-1=(t-1)\left(1+t+t^{2}+\ldots+t^{\ell-1}\right)=0 \bmod p$, one has $\left(1+t+t^{2}+\ldots+t^{\ell-1}\right)=0 \bmod p$, and so by (3.1) we have $b^{\ell} \leftrightharpoons a^{\ell}$. As $\ell$ is a divisor of $p-1$, we also have $b^{p-1} \leftrightharpoons a^{\ell}$. Consequently

$$
\left[a^{\ell}, b\right]=1 \text {. }
$$

Theorem 3.2. If $\mathcal{H}=(G ; a, b)$ is a regular oriented hypermap with p (prime) hyperfaces, each of valency $n$, then $\mathcal{H}$ is isomorphic to one of the following hypermaps: 
(1) $\mathcal{C M}_{n, p, u, t}=(M(n, p, u, t) ; a, b)$ for some $u, t \in\{0,1, \ldots, n-1\}$ such that

$$
(t-1) u=0 \bmod n \text { and } t^{p}=1 \bmod n ;
$$

(2) $\mathcal{H}_{n, u, v}^{p, \ell, t, k}=\left(G_{n, u, v}^{p, \ell, t} ; a, b a^{k}\right) \quad$ (p odd prime) , for some $\ell \in\{2, \ldots, n\}$, $u, v \in\{0, \ldots, n-1\}, k \in\{0, \ldots, \ell-1\}$ and $t \in\{2, \ldots, p-1\}$ such that

(H1) $\operatorname{gcd}(p-1, n)=0 \bmod \ell$,

(H2) $t^{\ell}=1 \bmod p$ and $t^{i} \neq 1 \bmod p$ for $i \in\{1,2, \ldots, \ell-1\}$

(that is, $t$ has order $\ell$ in $\left.\mathbb{Z}_{p}^{*}=\mathbb{Z}_{p} \backslash\{0\}\right)$,

(H3) $u=0 \bmod \ell, v=1 \bmod \ell$ and

(H4) $(t-1) u+p(v-1)=0 \bmod n$.

Moreover, all these hypermaps $\mathcal{H}_{n, u, v}^{p, \ell, t, k}$ for $\ell, t, k, n, u, v$ satisfying the above conditions, have p hyperfaces of valency $n$, and different parameters $(\ell, t, k, u, v)$ correspond to non-isomorphic hypermaps with p hyperfaces of valency $n$.

Proof. Let $\mathcal{H}=(G ; a, b)$ be a regular oriented hypermap with $p$ (prime) hyperfaces and $\mathcal{P}=(P ; A, B)$ be its primer hypermap where $A=a \Pi$ and $B=b \Pi$. By Theorem 16 of [4], $\mathcal{P}=\mathcal{P}_{k}^{p, \ell, t}$, for some $k, \ell$ and $t$. We treat separately the following cases: $\mathrm{A}=1$ (Case 1), $A \neq 1$ and $|B|=p($ Case 2$)$ and $A \neq 1$ and $|B| \neq p$ (Case 3).

Case 1. If $A=1$ then $\mathcal{H}$ is canonical metacyclic and $\mathcal{P}=\overline{\mathcal{P}}$ is the spherical cyclic hypermap $\mathcal{C}_{p}=\left(C_{p} ; 1, B\right)$ (Proposition 4 of [4]). Then $\mathcal{H}$ is isomorphic to $\mathcal{C M}_{n, p, u, t}=$ $(M(n, p, u, t) ; a, b)$ for some $u$ and $t$ such that $(t-1) u=0 \bmod n$ and $t^{p}=1 \bmod n$.

Case 2. $|A|=\ell>1$ and $|B|=p$. By Theorem 16 of [4], and Corollary 17 of [4] (see also $\S 1.2), \mathcal{P}(\mathcal{H})=\mathcal{P}_{0}^{p, \ell, t}=(P ; A, B)$, where

$$
P=\left\langle A, B \mid A^{\ell}=1, B^{p}=1, A^{-1} B A=B^{t}\right\rangle,
$$

for some $t \in\{1, \ldots, p-1\}$ such that $t^{\ell}=1 \bmod p$ and $t^{i} \neq 1 \bmod p$ for $i=$ $1,2, \ldots, \ell-1$. By Proposition 15 of [4], $\ell$ is a divisor of $p-1($ that is, $p=1 \bmod \ell)$.

From $A^{-1} B A=B^{t}$ we deduce that $B^{i} A B^{-i t}=A$ for each integer $i$. Applying Corollary 7 of [4] (see also $\S 1.2$ ), we derive the following relations in $G$ :

(i) $a^{n}=1$ with $n=0 \bmod \ell$;

(ii) $b^{p}=a^{u}, u=0 \bmod \ell$;

(iii) $\quad b^{i} a b^{-i t}=a^{v_{i}}, v_{i}=1 \bmod \ell, i=1, \ldots, p-1$ (also true for $i=p$ ).

These equations define a group that is right factorised by $K=\langle a\rangle$ into $p$ cosets. Indeed, on the one hand $K, K b, \ldots, K b^{p-1}$ are distinct cosets because $p$ is the smallest positive integer such that $b^{p}$ belongs to $K=\langle A\rangle$, and on the other hand, since $\{0, t, 2 t, \ldots,(p-1) t\}$ is a complete set of residues modulo $p$, equation (iii) implies that the set of right cosets of $K$ in $G$ is $G / r K=\left\{K, K b, \ldots, K b^{p-1}\right\}$. Hence the monodromy group of $\mathcal{H}$ is given by

$$
G=\left\langle a, b \mid a^{n}=1, b^{p}=a^{u}, b^{i} a b^{-i t}=a^{v_{i}}, i=1, \ldots, p-1\right\rangle
$$


for some integers $n=0 \bmod \ell, u=0 \bmod \ell$ and $v_{i}=1 \bmod \ell, i=1, \ldots, p-1$. We now simplify this presentation.

From relation (iii) we have $b^{i} a b^{-i t} \in\left\langle a^{\ell}\right\rangle a$, valid for any $i$. By Lemma 3.1,

$$
\left[a^{\ell}, b\right]=1 \text {. }
$$

Adding the relation $\left[a^{\ell}, b\right]=1$ to the presentation of $G$, some relations of the previous presentation will turn out to be redundant. From (iii), $i=1$, we deduce $b^{2} a b^{-2 t}=$ $b\left(b a b^{-t}\right) b^{-t}=b a^{v_{1}} b^{-t}=a^{v_{1}-1} b a b^{-t}=a^{2 v_{1}-1}=a^{2\left(v_{1}-1\right)+1} ;$ and more generally

$$
b^{i} a b^{-i t}=a^{i\left(v_{1}-1\right)+1}, i=1, \ldots, p-1 \text { and } p .
$$

Thus all the relations in (iii) except the first one are redundant. Now for $i=p$ we also have $b^{p} a b^{-p t}=a^{(1-t) u+1}$; therefore $a^{(1-t) u+1}=a^{p\left(v_{1}-1\right)+1}$ which implies

$$
(1-t) u=p\left(v_{1}-1\right) \bmod n .
$$

The hypermap $\mathcal{H}$ is then isomorphic to $\mathcal{H}_{n, u, v}^{p, \ell, t, 0}:=\left(G_{n, u, v}^{p, \ell, t} ; a, b\right)$, where

$$
G_{n, u, v}^{p, \ell, t}=\left\langle a, b \mid a^{n}=1, b^{p}=a^{u},\left[a^{\ell}, b\right]=1, b a b^{-t}=a^{v}\right\rangle,
$$

for some $\ell, t, n, u$ and $v$ such that $p=1 \bmod \ell, t^{\ell}=1 \bmod p, t^{i} \neq 1 \bmod p$ for each $i \in\{1,2, \ldots, \ell-1\}, u=0 \bmod \ell, v=1 \bmod \ell, n=0 \bmod \ell$ and $(1-t) u=$ $p(v-1) \bmod n$.

Conversely, we show that if $\mathcal{H}=\mathcal{H}_{n, u, v}^{p, \ell, t, 0}$ for some $\ell, t, n, u, v$ satisfying the above conditions, then $\mathcal{H}$ has $p$ hyperfaces of valency $n$. Factoring $G=G_{n, u, v}^{p, \ell, t}$ by the normal subgroup $\left\langle a^{\ell}\right\rangle$ yields the monodromy group of the primer hypermap $\mathcal{P}=\mathcal{P}_{0}^{p, \ell, t}$ with $p$ hyperfaces of valency $\ell$. Then $\ell$ divides $|a|$ and so both $\mathcal{P}=\mathcal{H} /\left\langle a^{\ell}\right\rangle$ and $\mathcal{H}$ have the same number of hyperfaces, $p$.

As $\operatorname{gcd}(t-1, p)=1$ there exist integers $c$ and $d$ such that $c(t-1)+d p=1$. Then the assignment $a \mapsto a, b \mapsto a^{c(1-v)+d u}$ turn each of the relators of $G$ into the identity of $C_{n}=\left\langle a \mid a^{n}=1\right\rangle$ and so it defines an epimorphism from $G$ to the cyclic group $C_{n}$. This proves that the order of $a$ in $G$ is $n$. Consequently $\mathcal{H}$ has $p$ hyperfaces of valency $n$.

Case 3. $|A|=\ell>1$ and $|B| \neq p$. By Theorem 16 of [4], and Corollary 17 of [4],

$$
\mathcal{P}(\mathcal{H})=\mathcal{P}_{k}^{p, \ell, t}=\left(M(p, \ell, 0, t) ; A, \beta A^{k}\right)=R_{k}((M(p, \ell, 0, t) ; A, \beta))=R_{k}\left(\mathcal{P}_{0}^{p, \ell, t}\right)
$$

for some $k \in\{0, \ldots, \ell-1\}$ and $t \in\{1,2, \ldots, p-1\}$, where $M(p, \ell, 0, t)=\langle\beta, A| \beta^{p}=$ $\left.A^{\ell}=1, \beta^{A}=\beta^{t}\right\rangle=\langle\beta\rangle \rtimes\langle A\rangle$ and (1) $\ell$ is a divisor of $p-1$, (2) $t^{\ell}=1 \bmod p$ and (3) if $\ell>1, t^{i} \neq 1 \bmod p$ for each $i \in\{1,2, \ldots, \ell-1\}$.

Then $\mathcal{P}\left(R_{\ell-k}(\mathcal{H})\right)=\mathcal{P}_{0}^{p, \ell, t}$. By case $2, R_{\ell-k}(\mathcal{H})=\mathcal{H}_{n, u, v}^{p, \ell, t, 0}=\left(G_{n, u, v}^{p, \ell, t} ; a, b\right)$. Thus

$$
\mathcal{H}=\mathcal{H}_{n, u, v}^{p, \ell, t, k}:=R_{k}\left(\mathcal{H}_{n, u, v}^{p, \ell, t, 0}\right)=\left(G_{n, u, v}^{p, \ell, t} ; a, b a^{k}\right),
$$

for some $k \in\{0, \ldots, \ell-1\}$.

Finally we show that different parameters lead to different hypermaps. 
(1) If $\mathcal{C} \mathcal{M}_{n, p, u, t}=(M ; a, b) \cong \mathcal{C} \mathcal{M}_{n^{\prime}, p^{\prime}, u^{\prime}, t^{\prime}}=\left(M^{\prime} ; \alpha, \beta\right)$ then $p^{\prime}=p$ and $n^{\prime}=n$, since the number of hyperfaces and their valencies should be the same. Then it becomes obvious that we must have $u^{\prime}=u$ and $t^{\prime}=t$ since these parameters run from 0 to $n-1$.

(2) If $\mathcal{H}_{n, u, v}^{p, \ell, t, k} \cong \mathcal{H}_{n^{\prime}, u^{\prime}, v^{\prime}}^{p^{\prime}, \ell^{\prime}, t^{\prime}, k^{\prime}}$, then, as above, we must have $p^{\prime}=p$ and $n^{\prime}=n$. Looking at the primer hypermaps,

$$
\mathcal{P}\left(\mathcal{H}_{n, u, v}^{p, \ell, t, k}\right)=\mathcal{P}_{k}^{p, \ell, t} \cong \mathcal{P}\left(\mathcal{H}_{n, u^{\prime}, v^{\prime}}^{p, \ell^{\prime}, t^{\prime}, k^{\prime}}\right)=\mathcal{P}_{k^{\prime}}^{p, \ell, t^{\prime}},
$$

this forces $\ell^{\prime}=\ell, t^{\prime}=t$ and $k^{\prime}=k$ (Theorem 16 [4]). But then we have $\mathcal{H}_{n, u, v}^{p, \ell, t, k}=$ $R_{k}\left(\mathcal{H}_{n, u, v}^{p, \ell, t, 0}\right)$ and $\mathcal{H}_{n, u^{\prime}, v^{\prime}}^{p, \ell, t, k}=R_{k}\left(\mathcal{H}_{n, u^{\prime}, v^{\prime}}^{p, \ell, t, 0}\right)$, so we must also have $\mathcal{H}_{n, u, v}^{p, \ell, t, 0} \cong \mathcal{H}_{n, u^{\prime}, v^{\prime}}^{p, \ell, t,}$. It is now clear that this isomorphism implies $u^{\prime}=u$ and $v^{\prime}=v$, since $u, u^{\prime}, v, v^{\prime}$ are restrict to $\{0, \ldots, n-1\}$.

Each hypermap $\mathcal{H}$ with $p$ hyperfaces, $p$ prime, with each hyperface of valency $n$, covers only one primer hypermap also with $p$ hyperfaces of valency $\ell=|A|$ (a divisor of $n$ ). This primer hypermap is

$$
\begin{aligned}
& \mathcal{P}\left(\mathcal{C M}_{n, p, u, t}\right)=\mathcal{C}_{p}=\left(C_{p} ; 1, B\right), \text { if } \mathcal{H}=\mathcal{C} \mathcal{M}_{n, p, u, t}, \text { or } \\
& \mathcal{P}\left(\mathcal{H}_{n, u, v}^{p, \ell, t, k}\right)=\mathcal{P}_{k}^{p, \ell, t}=\left(G_{0}^{p, \ell, t} ; a, b a^{k}\right), \text { if } \mathcal{H}=\mathcal{H}_{n, u, v}^{p, \ell, t, k}
\end{aligned}
$$

Among the hypermaps in the family $\mathcal{H}_{n, u, v}^{p, \ell, t, k}$ many of them share the same group and are distinguished by different pairs of generators. In the previous proof we find the requisites necessary to prove that $G_{n, u, v}^{p, \ell, t}$ is a metacyclic group.

Proposition 3.3. $G_{n, u, v}^{p, \ell, t}$ is a metacyclic group isomorphic to $G_{n, 0,1}^{p, \ell, t}=M(p, n, 0, t)=$ $\left\langle\beta, \alpha \mid \beta^{p}=1, \alpha^{n}=1, \alpha^{-1} \beta \alpha=\beta^{t}\right\rangle$ under the isomorphism $\psi: a \mapsto \alpha, b \mapsto \beta \alpha^{\theta}$, where $\theta=c(1-v)+d u$, for some $c$, d satisfying $c(t-1)+d p=1=\operatorname{gcd}(t-1, p)$. Moreover, $\mathcal{H}_{n, u, v}^{p, \ell, t, k} \cong R_{\theta+k}\left(\mathcal{H}_{n}^{p, \ell, t}\right)$, where $\mathcal{H}_{n}^{p, \ell, t}$ is the canonical metacyclic hypermap $\left(G_{n, 0,1}^{p, \ell, t} ; \alpha, \beta\right)$.

Proof. Consider the group

$$
\begin{aligned}
G_{n, 0,1}^{p, \ell, t} & =\left\langle\alpha, \beta \mid \alpha^{n}=1, \beta^{p}=1, \beta \alpha \beta^{-t}=\alpha\right\rangle \\
& =\left\langle\beta, \alpha \mid \beta^{p}=1, \alpha^{n}=1, \alpha^{-1} \beta \alpha=\beta^{t}\right\rangle \\
& =M(p, n, 0, t) .
\end{aligned}
$$

Note that $\beta \alpha \beta^{-t}=\alpha \Leftrightarrow \alpha^{-1} \beta \alpha=\beta^{t}$ implies that $\beta^{i} \alpha \beta^{-i t}=\alpha$ for any $i$; so by Lemma $3.1,\left[\alpha^{\ell}, \beta\right]=1$. This group, being metacyclic, has order $n p$. Note also that the condition $t^{\ell}=1 \bmod p$ is stronger than the metacyclic condition $t^{n}=1 \bmod p$. Let $c$ and $d$ be such that $c(t-1)+d p=1$, and let $\theta=c(1-v)+d u$. Then

$$
\begin{aligned}
& \theta=0 \bmod \ell, \text { so } \alpha^{\theta} \in Z\left(G_{n, 0,1}^{p, \ell, t}\right) \\
& \begin{aligned}
& \theta p=c p(1-v)+p d u=c(t-1) u+p d u=(c(t-1)+p d) u=u \quad, \quad \text { and } \\
& \theta(1-t) \quad=(1-t) c(1-v)+(1-t) u d \\
&=(1-t) c(1-v)+p(v-1) d \\
&=(t-1) c(v-1)+p(v-1) d \\
&=(v-1)((t-1) c+p d) \\
&=v-1 .
\end{aligned}
\end{aligned}
$$


The assignment $\psi: a \mapsto \alpha, b \mapsto \beta \alpha^{\theta}$, transfers the relators of $G_{n, u, v}^{p, \ell, t}$ to relators of $G_{n, 0,1}^{p, \ell, t}$ as we can observe:

1) $a^{n} \longrightarrow \alpha^{n}=1$,

2) $b^{p} a^{-u} \longrightarrow\left(\beta \alpha^{\theta}\right)^{p} \alpha^{-u}=\beta^{p} \alpha^{\theta p} \alpha^{-u}=\alpha^{u} \alpha^{-u}=1$,

3) $\left[a^{\ell}, b\right] \longrightarrow\left[\alpha^{\ell}, \beta \alpha^{\theta}\right]=\left[\alpha^{\ell}, \beta\right]=1$,

4) $b a b^{-t} a^{-v} \longrightarrow \beta \alpha^{\theta} \alpha\left(\beta \alpha^{\theta}\right)^{-t} \alpha^{-v}=\beta \alpha \beta^{-t} \alpha^{\theta} \alpha^{-\theta t} \alpha^{-v}$ $=\alpha^{\theta(1-t)} \alpha \alpha^{-v}=\alpha^{v-1} \alpha^{1-v}=1$,

By the Substitution Test [15, Theorem 4, pg 29], $\psi: G_{n, u, v}^{p, \ell, t} \rightarrow G_{n, 0,1}^{p, \ell, t}$ is an epimorphism. As $\left|G_{n, 0,1}^{p, \ell, t}\right|=\left|G_{n, u, v}^{p, \ell, t}\right|, \psi$ is indeed an isomorphism and thus $G_{n, u, v}^{p, \ell, t}$ is metacyclic. This isomorphism also shows that $\mathcal{H}_{n, u, v}^{p, \ell, t}=\mathcal{H}_{n, u, v}^{p, \ell, t, 0}$ is isomorphic to $R_{\theta}\left(\mathcal{H}_{n}^{p, \ell, t}\right)$. Then $\mathcal{H}_{n, u, v}^{p, \ell, t, k}=R_{k}\left(\mathcal{H}_{n, u, v}^{p, \ell, t}\right) \cong R_{\theta+k}\left(\mathcal{H}_{n}^{p, \ell, t}\right)$.

Corollary 3.4. If $\mathcal{H}$ is a regular oriented hypermap with a prime number of hyperfaces then its automorphism group is metacyclic, though $\mathcal{H}$ is not necessarily canonical metacyclic.

\section{Chirality groups and H-sequences}

Theorem 4.1. The chirality groups of $\mathcal{C M}_{n, p, u, t}$ and $\mathcal{H}_{n, u, v}^{p, \ell, t, k}$ are the cyclic groups $\left\langle a^{t^{2}-1}\right\rangle$ and $\left\langle b^{t^{2}-1}\right\rangle$ respectively. The chirality index of $\mathcal{C M}_{n, p, u, t}$ is $\frac{n}{\left(n, t^{2}-1\right)}$ while the chirality index of $\mathcal{H}_{n, u, v}^{p, \ell, t, k}$ is

$$
\frac{p}{\operatorname{gcd}\left(p, t^{2}-1\right)}= \begin{cases}1, & t=p-1 \\ p, & t \in\{2, \ldots, p-2\}\end{cases}
$$

Proof. The canonical metacyclic hypermap $\mathcal{C} \mathcal{M}_{n, p, u, t}$ has chirality group $\left\langle a^{t^{2}-1}\right\rangle$ [7].

By Proposition 3.3, $\mathcal{H}_{n, u, v}^{p, \ell, t, k}=R_{\theta+k}\left(\mathcal{H}_{n}^{p, \ell, t}\right)$ and, by Proposition 2.3, $\mathcal{H}_{n, u, v}^{p, \ell, t, k}$ has the same chirality group as the vertex-canonical metacyclic hypermap $\mathcal{H}_{n}^{p, \ell, t}=\left(G_{n}^{p, \ell, t} ; a, b\right)$, where

$$
G_{n}^{p, \ell, t}=M(p, n, 0, t)=\left\langle b, a \mid b^{p}=1, a^{n}=1, a^{-1} b a=b^{t}\right\rangle .
$$

Hence $\mathcal{H}_{n, u, v}^{p, \ell, t, k}$ has chirality group $\left\langle b^{t^{2}-1}\right\rangle$ [7], a subgroup of the cyclic group $\langle b\rangle$ of order $p$ (prime). If $\mathcal{H}_{n, u, v}^{p, \ell, t, k}$ is not reflexible $\left(b^{t^{2}-1} \neq 1 \Leftrightarrow t \neq-1 \bmod p\right.$, since $\left.t \geq 2\right)$, it must has order $p$, and thus $\mathcal{H}_{n, u, v}^{p, \ell, t, k}$ has chirality index $\kappa=p$.

Corollary 4.2. If $\mathcal{H}$ is a regular oriented hypermap with a prime number of hyperfaces and its primer hypermap is $\mathcal{P}=(P ; A, B)$, with $A \neq 1$, then $\mathcal{H}$ is reflexible if and only if $|A|=2$. 
Theorem 4.3. The H-sequences of the hypermaps of Theorem 3.2 are

$$
\begin{aligned}
& \mathcal{C M}_{n, p, u, t}: {\left[\frac{p n}{(n, u)}, \frac{p n}{\left(n, t^{p-1}+\ldots+1+u\right)}, n ;(n, u),\left(n, t^{p-1}+\ldots+1+u\right), p ; p n\right] } \\
& \mathcal{H}_{n, u, v}^{p, \ell, t, 0}: {\left[\frac{p n}{(n, u)}, \frac{n}{(n, \theta+1)}, n ;(n, u), p(n, \theta+1), p ; p n\right] } \\
& \mathcal{H}_{n, u, v}^{p, \ell, t, \ell-1}: {\left[\frac{n}{(n, \theta+\ell-1)},\left(\left(\frac{p n}{(n, u)}, \frac{n}{\ell}\right)\right), n ; p(n, \theta+\ell-1), \frac{p n}{\left(\left(\frac{p n}{(n, u)}, \frac{n}{\ell}\right)\right)}, p ; p n\right] } \\
& \mathcal{H}_{n, u, v}^{p, \ell, t, k}: {\left[\frac{n}{(n, \theta+k)}, \frac{n}{(n, \theta+k+1)}, n ; p(n, \theta+k), p(n, \theta+k+1), p ; p n\right], } \\
&(0<k<\ell-1)
\end{aligned}
$$

where, for space saving, $(u, v)$ stands for $\operatorname{gcd}(u, v),((u, v))$ stands for $\operatorname{lcm}(u, v)$, the least common multiple of $u$ and $v, \theta=c(1-v)+d u$, and $c$ and $d$ are integers such that $c(t-1)+d p=1=(t-1, p)$.

Proof. Recall that the H-sequence of $\mathcal{H}=(G ; a, b)$ is a sequence of numbers

$$
[|b|,|a b|,|a| ; V, E, F ;|G|]
$$

where $|b|$ (the order of $b$ ) is the hypervertex-valency, $|a b|$ is the hyperedge-valency, $|a|$ is the hyperface-valency, $V=\frac{|G|}{|b|}$ is the number of hypervertices, $E=\frac{|G|}{|a b|}$ is the number of hyperedges and $F=\frac{|G|}{|a|}$ is the number of hyperfaces.

- For the canonical metacyclic hypermap $\mathcal{C M}_{n, p, u, t}=(G ; a, b)$, where

$$
G=M(n, p, u, t)=\left\langle a, b \mid a^{n}=1, b^{p}=a^{u}, b^{-1} a b=a^{t}\right\rangle,
$$

it is clear that $|a|=n,|b|=\frac{p n}{\operatorname{gcd}(n, u)}$ and $|G|=p n$. We only need to calculate $|a b|=|b a|$. From the relation $b^{-1} a b=a^{t}$ we get: $a^{m} b=b a^{m t}$ and $a b^{m}=b^{m} a^{t^{m}}$, for any positive integer $m$. From this we easily derive

$$
(b a)^{m}=b^{m} a^{t^{m-1}+\cdots+t+1} .
$$

Now as $\mathcal{C} \mathcal{M}_{n, p, u, t}$ covers $\mathcal{P}=\left(C_{p} ; 1, B\right)$, the order of $b a$ is a multiple of $p$. As $(b a)^{p}=a^{t^{p-1}+\cdots+t+1+u}$, then $|b a|=\frac{p n}{\operatorname{gcd}\left(n, t^{p-1}+\ldots+1+u\right)}$. The rest of the Hsequence is easily determined from these values.

- For $\mathcal{H}=\mathcal{H}_{n, u, v}^{p, \ell, t, 0}=(G ; a, b)$, where $G=G_{n, u, v}^{p, \ell, t}$, we also have $|a|=n,|b|=$ $\frac{p n}{\operatorname{gcd}(n, u)}$ and by Proposition 3.3, $|G|=p n$. Therefore we only need to calculate $|a b|=|b a|$.

- For $\mathcal{H}=\mathcal{H}_{n, u, v}^{p, \ell, t, \ell-1}=\left(G ; a, b a^{\ell-1}\right)$, where $G=G_{n, u, v}^{p, \ell, t}$, we have $|a|=n$, $\left|a b a^{\ell-1}\right|=\left|b a^{\ell}\right|=\operatorname{lcm}\left(|b|,\left|a^{\ell}\right|\right)=\operatorname{lcm}\left(\frac{p n}{\operatorname{gcd}(n, u)}, \frac{n}{\ell}\right)$, where $|G|=p n$. Therefore we only need to calculate $\left|b a^{\ell-1}\right|$.

- For $\mathcal{H}=\mathcal{H}_{n, u, v}^{p, \ell, t, k}=\left(G ; a, b a^{k}\right)$, where $G=G_{n, u, v}^{p, \ell, t}$, we have $|a|=n,\left|a b a^{k}\right|=$ $\left|b a^{k+1}\right|$ and $|G|=p n$. Therefore we only need to calculate $\left|b a^{k+1}\right|$. 
To complete the $\mathrm{H}$-sequence in the last three cases, we need to calculate the order of $a^{j} b$ for $j \in\{1, \ldots, \ell-1\}$, within the group $G=G_{n, u, v}^{p, \ell, t}$. This group is isomorphic to the metacyclic group $M(p, n, 0, t)=\left\langle\beta, \alpha \mid \beta^{p}=1, \alpha^{n}=1, \beta^{\alpha}=\beta^{t}\right\rangle$ under the isomorphism $\psi: G \rightarrow M(p, n, 0, t), a \mapsto \alpha, b \mapsto \beta \alpha^{\theta}$ (Proposition 3.3), where $\theta=$ $c(1-v)+d u$ (which is a multiple of $\ell$ ), and $c$ and $d$ are integers such that $c(t-1)+d p=1$. Then the order of $a^{j} b$ is the order of $\alpha^{j} \beta \alpha^{\theta}=\alpha^{\theta+j} \beta=\alpha^{i} \beta$, where $i=\theta+j \neq 0 \bmod \ell$. We now follow the proof of Corollary 17 of [4] to compute the order of $\beta \alpha^{i}$.

The third equation of $M(p, n, 0, t)$ implies that $\beta^{\alpha^{i}}=\beta^{t^{i}} \Leftrightarrow \beta \alpha^{i}=\alpha^{i} \beta^{t^{i}}$. By induction we get

$$
\left(\beta \alpha^{i}\right)^{m}=\alpha^{i m} \beta^{t^{i m}+t^{i(m-1)}+\cdots+t^{i}}=\alpha^{i m} \beta^{V(m)},
$$

where $V(m)=t^{i m}+t^{i(m-1)}+\cdots+t^{i}=t^{i}\left(t^{i(m-1)}+\cdots+t+1\right)$. Let $U(m)=$ $t^{i(m-1)}+\cdots+t+1$. Now the order of $\beta \alpha^{i}$ is the least positive integer $m$ such that

$$
\left(\beta \alpha^{i}\right)^{m}=1 \Leftrightarrow \alpha^{i m} \beta^{V(m)}=1 \Leftrightarrow \alpha^{i m}=\beta^{-V(m)} \in\langle\alpha\rangle \cap\langle\beta\rangle=1 \Leftrightarrow \alpha^{i m}=\beta^{V(m)}=1 .
$$

Hence $m$ is multiple of $\left|\alpha^{i}\right|$. On the other hand, $\beta$ has order $p$ and $\beta^{V(m)}=1 \Leftrightarrow V(m)=$ $0 \bmod p \Leftrightarrow t^{i} U(m)=0 \bmod p \Leftrightarrow U(m)=0 \bmod p$, since $t \in \mathbb{Z}_{p}^{*}=\{1,2, \ldots, p-1\}$. Now $\ell \mid p-1$ and $t^{\ell}=1 \bmod p$ and $t^{q} \neq 1 \bmod p$, for any $q<\ell$, that is $t^{q} \neq 1 \bmod p$ for any $q \neq 0 \bmod \ell$. Since $i=\theta+j \neq 0 \bmod \ell$, then $U(m)=\frac{t^{i m}-1}{t^{i}-1}$, and so

$$
U(m)=0 \bmod p \Leftrightarrow t^{i m}=1 \bmod p .
$$

Thus, if $m$ is just the order of $\alpha^{i}$, that is, if $m=\frac{n}{\operatorname{gcd}(n, i)}$, then $i m$ is a multiple of $n$ and so a multiple of $\ell(n=0 \bmod \ell)$, and consequently $m$ also satisfies $\beta^{V(m)}=1$. Hence $\left|\beta \alpha^{i}\right|=\left|\alpha^{i}\right|$, that is,

$$
\left|a^{j} b\right|=\left|\beta \alpha^{\theta+j}\right|=\left|\alpha^{\theta+j}\right|=\frac{n}{\operatorname{gcd}(n, \theta+j)} .
$$

\section{Number of hypermaps with $p$ hyperfaces}

To count the number of regular oriented hypermaps with $p$ (prime) hyperfaces of valency $n$ it suffices to count the different parameters appearing in items (1) and (2) of Theorem 3.2. Let $N H_{(1)}(p, n)$ be the number of regular oriented hypermaps $\mathcal{C M}_{n, p, u, t}$ in item (1) with $p$ hyperfaces of valency $n$, and $\mathrm{NH}_{(2)}(p, n)$ be the number of regular oriented hypermaps $\mathcal{H}_{n, u, v}^{p, \ell, t, k}$ in item (2) with $p$ hyperfaces of valency $n$. Then

(1) Denote by $U_{p}(n)$ the subgroup of the units of $\mathbb{Z}_{n}$ whose elements $t$ satisfy $t^{p}=$ $1 \bmod n$. Let $\mu(t)$ be the number of solutions $u$ of $(t-1) u=0 \bmod n$. Then

$$
N H_{(1)}(p, n)=\sum_{t \in U_{p}(n)} \mu(t)=\sum_{t \in U_{p}(n)} \operatorname{gcd}(t-1, n) .
$$

Now let $N R H_{(1)}(p, n)$ and $N C H_{(1)}(p, n)$ be the number of reflexible and chiral (respectively) regular oriented canonical metacyclic hypermaps $\mathcal{H}=\mathcal{C} \mathcal{M}_{n, p, u, t}$ in 
item (1) with $p$ hyperfaces of valency $n$. By Theorem 4.1, $\mathcal{H}$ is reflexible if and only if $t^{2}=1 \bmod n$. This implies that $t^{m}=1 \bmod n$ for any even $m$, and so, combining with $t^{p}=1 \bmod n$, we get $t=1 \bmod n$. Then

$$
N R H_{(1)}(p, n)=\operatorname{gcd}(0, n)=n
$$

and

$$
N C H_{(1)}(p, n)=\sum_{t \in U_{p}^{*}(n)} \operatorname{gcd}(t-1, n),
$$

where $U_{p}^{*}(n)=\left\{t \in U_{p}(n) \mid t \neq 1 \bmod n\right\}$.

(2) Denote by $\wp(t, \ell)$ the number of pairs $(u, v)$ satisfying the equations $u=0 \bmod \ell$, $v-1=0 \bmod \ell$ and $(H 4)$. Since $k$ freely ranges in $\{0,1, \ldots, \ell-1\}$, then

$$
N H_{(2)}(p, n)=\sum_{\substack{\ell \mid \operatorname{gcd}(p-1, n) \\ \ell>1}} \sum_{t \in G_{\ell}} \sum_{k} \wp(t, \ell)=\sum_{\substack{\ell \mid \operatorname{gcd}(p-1, n) \\ \ell>1}} \sum_{t \in G_{\ell}} \ell \wp(t, \ell),
$$

where $G_{\ell}$ is the set of elements of order $\ell$ in the cyclic group $\mathbb{Z}_{p}{ }^{*}=C_{p-1}$. Since $p$ and $t-1$ are coprimes, the number of pairs of solutions $(u, v-1) \bmod n$ of $(H 4)$ is exactly $n$; so the number $\wp(t, \ell)$ of solutions pairs $(u, v-1) \bmod n$ which are multiple of $\ell$, where $n=0 \bmod \ell$, of $(H 4)$, is the number of solutions pairs $\left(\frac{u}{\ell}, \frac{v-1}{\ell}\right) \bmod \frac{n}{\ell}$ of

$$
\frac{u}{\ell}(t-1)+\frac{v-1}{\ell} p=0 \bmod \frac{n}{\ell},
$$

which is exactly $\wp(t, \ell)=\frac{n}{\ell}$. Therefore

$$
N H_{(2)}(p, n)=\sum_{\substack{\ell \operatorname{gcd}(p-1, n) \\ \ell>1}} \sum_{t \in G_{\ell}} \ell \frac{n}{\ell}=n \sum_{\substack{\ell \mid \operatorname{gcd}(p-1, n) \\ \ell>1}} \Phi(\ell),
$$

where $\Phi$ is the Euler Phi-function. In the special case when $p$ is a Fermat prime, $p-1$ is a power of 2 and so $N H_{(2)}(p, n)=0$ for $n$ odd. The total number $N H(p, n)$ of regular oriented hypermaps with $p$ (prime) hyperfaces of valency $n$ is then given by:

$$
\begin{aligned}
N H(p, n) & =N H_{(1)}(p, n)+N H_{(2)}(p, n) \\
& =\sum_{t \in U_{p}(n)} \operatorname{gcd}(t-1, n)+\sum_{\substack{\ell \mid \operatorname{gcd}(p-1, n) \\
\ell>1}} n \Phi(\ell) .
\end{aligned}
$$

Denote by $\mathrm{NRH}_{(2)}(p, n)$ and $\mathrm{NCH}_{(2)}(p, n)$ the number of reflexible and chiral (respectively) regular oriented hypermaps $\mathcal{H}=\mathcal{H}_{n, u, v}^{p, \ell, t, k}$ in item (2). By Theorem 4.1, $\mathcal{H}$ is reflexible if and only if $t=-1 \bmod p$. This is equivalent to $\ell=2$ (and this 
implies $n$ even). Hence

$$
\begin{aligned}
& \operatorname{NRH}_{(2)}(p, n)= \begin{cases}0, & \text { if } n \text { is odd, } \\
n, & \text { if } n \text { is even. }\end{cases} \\
& N C H_{(2)}(p, n)=n \sum_{\substack{\ell \mid \operatorname{gcd}(p-1, n) \\
\ell>2}} \Phi(\ell) .
\end{aligned}
$$

Note that if $n$ is odd then $\mathcal{H}_{n, u, v}^{p, \ell, t, k}$ is chiral with chirality index $p$.

Denoting by $N R H(p, n)$ and $N C H(p, n)$ the number of reflexible and chiral regular oriented hypermaps with $p$ (prime) hyperfaces of valency $n$, then

$$
N R H(p, n)=N R H_{(1)}(p, n)+N R H_{(2)}(p, n)= \begin{cases}n, & \text { if } n \text { is odd } \\ 2 n, & \text { if } n \text { is even }\end{cases}
$$

and $N C H(p, n)=N C H_{(1)}(p, n)+N C H_{(2)}(p, n)$.

\section{References}

[1] G. V. Bely̆̌, Galois extensions of a maximal cyclotomic field, Izv. Akad. Nauk SSSR Ser. Mat. 43 (1979), 267-276, 479.

[2] A. Breda, A. Breda D'Azevedo and R. Nedela, Chirality group and chirality index of Coxeter chiral maps, Ars Combin. 81 (2006), 147-160.

[3] A. Breda d'Azevedo and M. E. Fernandes, Regular oriented hypermaps up to five hyperfaces, Acta Univ. M. Belii Ser. Math. 17 (2010), 21-39.

[4] A. Breda D'Azevedo and M. E. Fernandes, Classification of primer hypermaps with a prime number of hyperfaces, European J. Combin. 32 (2011), 233-242, doi:10.1016/j.ejc.2010.09. 003.

[5] A. Breda D’Azevedo, G. Jones, R. Nedela and M. Škoviera, Chirality groups of maps and hypermaps, J. Algebraic Combin. 29 (2009), 337-355, doi:10.1007/s10801-008-0138-z.

[6] A. Breda D'Azevedo and G. A. Jones, Totally chiral maps and hypermaps of small genus, $J$. Algebra 322 (2009), 3971-3996, doi:10.1016/j.jalgebra.2009.02.017.

[7] A. Breda D'Azevedo and R. Nedela, Chiral hypermaps with few hyperfaces, Math. Slovaca 53 (2003), 107-128.

[8] A. Breda d'Azevedo, I. I. Rodrigues and M. E. Fernandes, On chirality groups and regular coverings of regular oriented hypermaps, Czechoslovak Math. J. 61(136) (2011), 1037-1047, doi:10.1007/s10587-011-0046-6.

[9] A. J. Breda d'Azevedo, The reflexible hypermaps of characteristic -2, Math. Slovaca 47 (1997), 131-153.

[10] R. Cori, Un code pour les graphes planaires et ses applications, Société Mathématique de France, Paris, 1975, with an English abstract, Astérisque, No. 27.

[11] D. Corn and D. Singerman, Regular hypermaps, European J. Combin. 9 (1988), 337-351, doi: 10.1016/S0195-6698(88)80064-7. 
[12] S.-F. Du, J. H. Kwak and R. Nedela, A classification of regular embeddings of graphs of order a product of two primes, J. Algebraic Combin. 19 (2004), 123-141, doi:10.1023/B: JACO.0000023003.69690.18.

[13] S.-F. Du, J. H. Kwak and R. Nedela, Regular embeddings of complete multipartite graphs, European J. Combin. 26 (2005), 505-519, doi:10.1016/j.ejc.2004.02.010.

[14] A. Grothendieck, Equisse d'un programme (1984), in: P. Lochak and L. Schneps (eds.), Geometric Galois actions, vol. 2, Cambridge Univ. Press, Cambridge, volume 242 of London Math. Soc. Lecture Note Ser., pp. 243-284, 1997, doi:10.1017/CBO9780511666124.014.

[15] D. L. Johnson, Topics in the theory of group presentations, volume 42 of London Mathematical Society Lecture Note Series, Cambridge University Press, Cambridge-New York, 1980.

[16] G. Jones and D. Singerman, Maps, hypermaps and triangle groups, in: The Grothendieck theory of dessins d'enfants (Luminy, 1993), Cambridge Univ. Press, Cambridge, volume 200 of London Math. Soc. Lecture Note Ser., pp. 115-145, 1994.

[17] G. A. Jones and D. Singerman, Theory of maps on orientable surfaces, Proc. London Math. Soc. (3) 37 (1978), 273-307.

[18] T. R. S. Walsh, Hypermaps versus bipartite maps, J. Combinatorial Theory Ser. B 18 (1975), $155-163$.

[19] S. Wilson and A. Breda d'Azevedo, Non-orientable maps and hypermaps with few faces, $J$. Geom. Graph. 7 (2003), 173-189. 